\title{
Detección neonatal en el Paraguay. Brechas para su cobertura universal
}

\author{
Marta Ascurra', Edgar Giménez Caballero ${ }^{I I}$, Margarita Samudio III \\ I. Programa de Prevención de la Fibrosis Quística y Retardo Mental. Ministerio de Salud Pública y Bienestar \\ Social \\ II. Instituto Desarrollo. Área de salud pública \\ III. Instituto de Investigaciones de Ciencias de la Salud. Universidad Nacional de Asunción
}

\section{Cómo referenciar este artículo/} How to reference this article:
Ascurra M, Giménez E, Samudio M. Detección neonatal en el Paraguay. Brechas para su cobertura universal. Mem. Inst. Investig. Cienc. Salud. 2015;13(2):86-102

\section{R E S U M E N}

La cobertura universal en los programas de detección neonatal (DN) es una obligación de todos los países, de manera a ofrecer a la población los servicios de detección y tratamiento oportunos sin necesidad de que la misma incurra en gastos considerables ni pago directo al momento de recibir el servicio. El objetivo de este estudio fue determinar las brechas para la universalización de la cobertura de la DN en Paraguay. La unidad de análisis fue el Programa de Prevención de la Fibrosis Quística y Retardo Mental (PPFQRM). Se evaluaron los tres ejes de la cobertura universal sanitaria (CUS) propuesto por la OMS: amplitud o proporción cubierta, profundidad definida por el número de patologías incluidas en la DN, nivel de protección financiera; así como las brechas para el logro de la universalización de la cobertura de la DN. La información se obtuvo del PPFQRM, Dirección General de Estadísticas Vitales y de la Dirección General de Estadística, Encuesta y Censo. Se encontró que los estudios de diagnóstico y tratamiento están garantizados por la Ley $2138 / 2003$. La amplitud tuvo un aumento significativo de $0,8 \%$ en 1999 a 76,1\% en 2.014. Existe necesidad de ampliar las patologías incluidas en la DN en el PPFQRM (hipotiroidismo congénito, fenilcetonuria y fibrosis quística). Se pudo comprobar los grandes esfuerzos e inversiones que el Paraguay está realizando para el logro de la universalización de la DN; con lo cual se contribuirá a romper el círculo de pobreza, considerando que la discapacidad genera pobreza y la pobreza discapacidad.

Palabras clave: Detección neonatal, cobertura universal, Paraguay, brechas.

\section{Neonatal screening in Paraguay. Gaps for universal coverage}

\section{A B S T R A C T}

Universal coverage in neonatal screening (NS) programs is an obligation of all countries, providing the population screening services and treatment without the population incurring considerable expenses or direct payment at the time of the service. The objective of this descriptive cross-sectional study was to determine the gaps in universal coverage of NS in Paraguay. The unit of analysis was the Program for Prevention of Cystic Fibrosis (PPFQRM). The three axes of universal health coverage (UHC) proposed by WHO were evaluated: amplitude or proportion of the population covered, depth or pathologies included in the ND, and financial protection level in addition to the gaps to achieve universal coverage of the program. Data were extracted from the PPFQRM database, Department of Vital Statistics and the General Direction of Statistics, Surveys and Census. It was found that both the diagnostic studies and treatment are guaranteed by the Law $2138 / 2003$. The amplitude had a significant increase of $0.8 \%$ in 1999 to $76.1 \%$ in 2014 . There is a need to ample the pathologies included (primary congenital hypothyroidism, 
phenylketonuria, cystic fibrosis) in the NS of the PPFQRM. It was found that Paraguay is making great efforts and investments towards achieving universal coverage in the NS. Achieving universal NS will contribute to break the cycle of poverty considering that disability causes poverty and disability poverty.

Key words: neonatal detection, universal coverage, Paraguay, gaps.

\section{INTRODUCCION}

La Cobertura Universal de Salud (CUS) es uno de los conceptos más poderosos de la salud pública para llevar a un ámbito concreto lo que se expresa como el derecho a la salud de la población. Esta universalización se entiende como la disponibilidad, acceso y utilización efectiva de servicios de salud integrales cuando se necesita sin que la persona o la familia tenga que incurrir en gastos excesivos o incluso correr riesgo de una ruina financiera o empobrecimiento a causa de ello (1).

Para valorar el grado de cobertura de los servicios de salud se distinguen tres dimensiones de análisis: la amplitud, la profundidad y el nivel de cobertura. La amplitud de la cobertura se refiere a la proporción de la población cubierta considerando las distintas estratificaciones poblaciones según criterios socioeconómicos que actúan como determinantes sociales de la salud. La profundidad de la cobertura se refiere al conjunto de servicios que garantiza la cobertura para las distintas poblaciones. En tanto que el nivel de la cobertura se refiere al grado de protección financiera de la población para acceder y utilizar dichos servicios. Entre los requisitos para contar con una Cobertura Universal de Salud, se describen un sistema de salud sólido, financieramente sustentable, con servicios de salud distribuidos con equidad para toda la población, con personal de salud capacitado, medicamentos esenciales y tecnología adecuada, ambos de alta calidad (2).

Los programas de detección neonatal (DN), forman parte esencial de todo servicio de salud que pretenda una atención integral a la primera infancia. Consisten en un sistema interdisciplinario para detectar precozmente enfermedades congénitas que pueden causar graves daños, incluso la muerte, en una población de recién nacidos y que son pasibles de ser evitadas a través del tratamiento dentro del primer mes de vida. La prevención, a través de la detección y tratamiento oportuno de las enfermedades, debe ser realizada por medio de métodos eficaces, técnicamente sencillos y accesibles para toda la población (3-5).

Con criterios de justicia social y equidad sanitaria es razonable sustentar que la cobertura de este servicio debería ser universal, es decir otorgar a todos los RN del país la detección y el tratamiento oportunos mediante la DN que incluya un conjunto de determinaciones relevantes para el contexto nacional y sin que las familias deban incurrir en gastos considerables para obtenerlas y evitando el pago directo al momento de recibir el servicio. Esta posición es coherente con el planteamiento de reestructuración del Sistema Nacional de Salud en redes integradas de servicios de salud basados en Atención Primaria de la Salud (APS) y en este marco, la búsqueda de la cobertura universal de salud (CUS) $(1,6)$.

En Paraguay, la DN está a cargo del Programa de Prevención de la Fibrosis Quística y del Retardo Mental, creado por la Ley 2138 en el 2.003 (7). Contempla al hipotiroidismo congénito $(\mathrm{HC})$, patología que causa grave retardo físico y mental y con alta incidencia en países mediterráneos y cuyo tratamiento es de muy bajo costo (7).

La fenilcetonuria (PKU) es de origen genético al igual que la fibrosis quística (FQ), pudiendo nacer en una familia más de un individuo afectado. En la PKU el recién nacido no detectado y tratado de forma precoz presenta un grave daño neurológico, que se presenta por la pérdida de habilidades ya adquiridas, autismo, convulsiones, las personas por lo general terminan siendo hospitalizadas en instituciones siquiátricas, el tratamiento consiste en la restricción de los niveles de fenilalanina (FA) (8). 
En las personas afectadas por la FQ se observa un daño a nivel de las glándulas de secreción; el sistema pulmonar y digestivo están principalmente afectados. La falta de detección y tratamiento no permite una buena calidad de vida, con una alta morbimortalidad $(9,10)$.

El presente estudio busca determinar la situación actual de la DN así como las brechas para la universalización de la cobertura de la DN en Paraguay considerando el alcance poblacional, el conjunto de servicios otorgados para la detección, el diagnóstico y tratamiento así como una aproximación a las brechas financieras para lograrlo.

\section{MATERIALES Y METODO}

El diseño del estudio corresponde a un estudio transversal descriptivo donde la unidad de análisis es el Programa de Prevención de la Fibrosis Quística y del Retardo Mental (PPFQRM).

Para determinar la situación actual del Programa que tiene a su cargo la DN en el Paraguay desde sus inicios hasta la actualidad, se ha llevado a cabo un análisis de la base de datos y publicaciones del PPFQRM y de su página web. Se analizaron la situación legal, los recursos humanos, la capacitación y difusión, los efectores para la recolección de muestras de sangre, las características de las muestras de sangre, el transporte utilizado para el traslado de las mismas, el laboratorio tanto de pesquisa como el de confirmación de las patologías, el tratamiento ofrecido las fuentes de financiamiento con que se cuenta así como las posibilidades de expansión a otras patologías.

El universo del estudio corresponde a todos los recién nacidos cuyas muestras hayan sido analizadas y que recibieron servicios en el PPFQRM, así como a las personas afectadas por la fibrosis quística.

La incidencia ha sido establecida solo para dos de las patologías, el hipotiroidismo congénito (HC) y la fenilcetonuria (PKU) para 2.013 y 2.014 y desde su origen hasta diciembre del 2.014. Los datos fueron extraídos de la base de datos del PPFQRM, así como de la Dirección General de Estadísticas Vitales del MSPyBS y Encuestas y la Dirección General de Encuestas, Estadísticas y Censo (DGEEC).

Los datos extraídos con anterioridad fueron utilizados para la identificación de los tres ejes o dimensiones de la cobertura según el modelo conceptual de la CUS propuesto por la OMS: a) amplitud o proporción cubierta por el PPFQRM, tanto para la detección como para el tratamiento), b) profundidad o conjunto de servicios de salud otorgados por el PPFQRM, en relación a sitios de atención como entrega de medicamentos entre otros insumos, y c) nivel o grado de protección financiera, costos cubiertos para recibir servicios de diagnóstico y tratamiento en el programa nacional; así como para identificar las brechas para la universalización de la cobertura del Programa. El nivel de análisis fue nacional y por región sanitaria (RS). Por último, se propondrán estrategias que permitan llegar a la universalización.

\section{RESULTADOS}

Al analizar los componentes del Programa de Detección Neonatal del Paraguay, hemos encontrado cuanto sigue:

Legislación: Se cuenta con la Ley 2138/2003, que crea el Programa de Prevención de la Fibrosis Quística y el Retardo Mental (PPFQRM) producido por el HC y la PKU, que cuenta con 7 Artículos, y que estipula que todas las instituciones públicas o privadas donde se producen nacimientos estarán obligadas a realizar los estudios a los RN para las tres patologías y los gastos estarán previstos en el Presupuesto General de la Nación. A su vez, el Artículo 7 menciona que los casos detectados serán tratados en el PPFQRM. También indica la responsabilidad de los progenitores para acercar al RN a un sitio de toma de muestra, en el caso de un parto domiciliario. La Resolución de la Secretaria General del Ministerio de Salud N02126 de abril del 2.004, incorporó al PPFQRM a la Red 
de Programas del MSPBS. El organigrama del Programa se observa en la Figura 1, también incluye la detección de los trastornos auditivos (11).

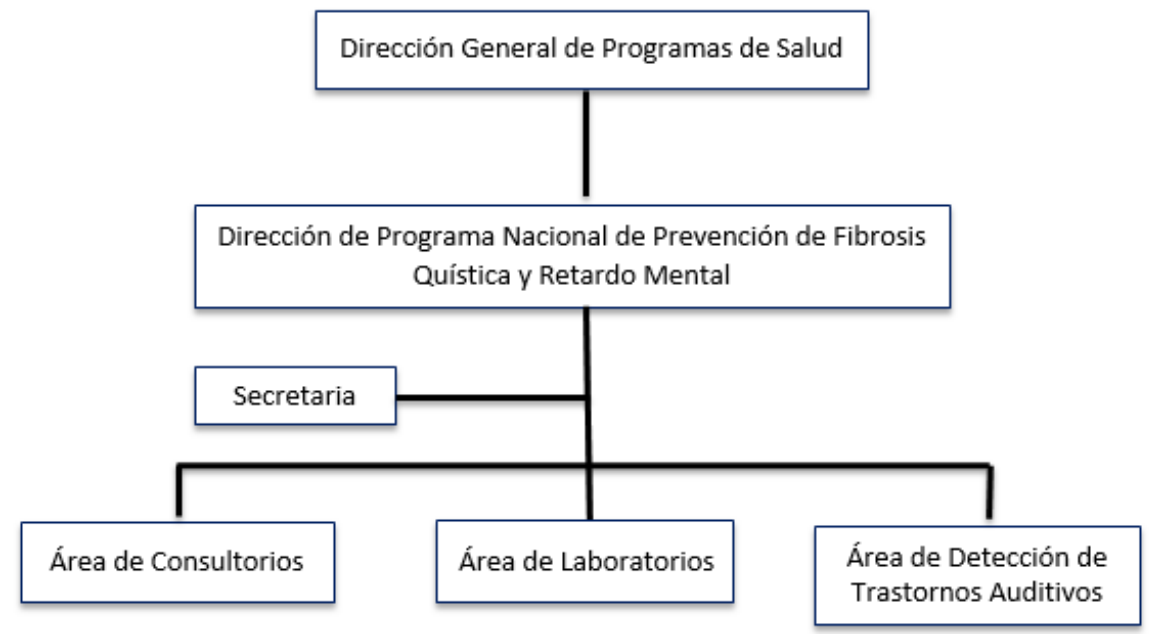

Figura 1. Organigrama actual del PPFQRM

Recursos humanos (RRHH): Los RRHH del programa se clasificaron en dos grupos, los que tenían tareas de DN propiamente dichas, que a su vez se separaron según la actividad que realizan en personal administrativo, del laboratorio, de consultorio y tratamiento, de informática, área de trasporte y limpieza; y los RRHH de los Sitios de Toma de Muestra (STM). Un STM es aquel donde se lleva a cabo la toma de muestra del RN y por lo general está a cargo de dos a tres personas para cubrir las guardias y los fines de semana.

A nivel central, en octubre del 1.999, se inició la DN con dos personas, al cual se incorporaron en el trascurso del tiempo cuatro más a nivel administrativo, tres bioquímicas para los estudios de tamizaje y confirmación, dos médicos especialistas y un kinesiólogo para el tratamiento de las patologías detectadas, dos personas para el área de informática, un chofer y un personal de limpieza, sumando en la actualidad un total de 16 personas. No se cuenta con área de asistencia social, esta tarea es desarrollada por personal del área administrativa, ni con personal para cubrir el área de Trastornos Auditivos (Tabla 1).

Tabla 1. Recursos Humanos a nivel central del PPFQRM y Retardo Mental

\begin{tabular}{lll}
\hline Área de desempeño & Funciones & $\mathbf{n}$ \\
\hline Directora del Programa & Coordinación de las acciones del PPFQRM & 1 \\
Administrador & Compra/entrega de medicamentos, reactivos e insumos & 1 \\
Secretaria/Jefe de RRHH & Tareas de secretaría/Apoyo al personal & 1 \\
Recepción de muestras & Recepción y codificación de muestras & 2 \\
Informática & Carga de datos & 2 \\
Laboratorista & Tamizaje & 4 \\
Médicos & Gastroenterólogo, Endocrinólogo & 2 \\
Kinesiólogo & Atención kinesiológica & 1 \\
Chofer & Búsqueda activa de pacientes & 1 \\
Personal de limpieza & Limpieza del Programa & 1 \\
\hline
\end{tabular}

Fuente: PPFQRM 
En octubre de 1.999, se inició el programa con un STM en una Región Sanitaria (RS), al cual se le fueron sumando luego los demás STM hasta llegar a 942 en diciembre del 2.014. El PPFQRM cuenta con un mínimo de 958 personas, si consideramos un encargado por sitio de toma de muestra más los miembros del Programa o 1.900 si por sitio hubiese dos encargadas. En la Figura 2, se ve el aumento gradual por año del número de STM así como la incorporación de RS.

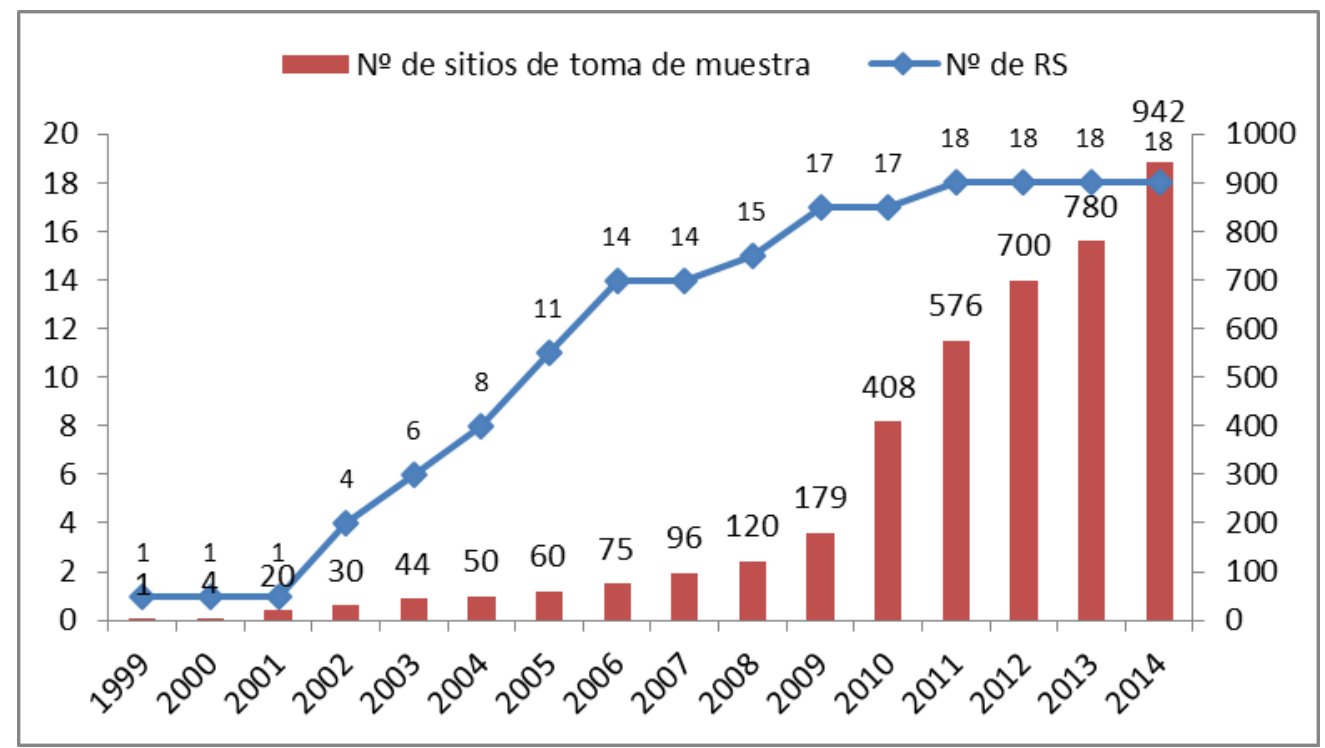

Fuente: Programa de Prevención de la Fibrosis Quística y Retardo Mental

Figura 2. No de STM incorporados por año y región sanitaria (RS)

Capacitación: A nivel central se contaban con un $73 \%$ de profesionales (11/15) en el área en el cual se desempeñan, de los cuales 33\% eran docentes investigadores. A su vez 40\% (6/15) fue capacitado en Japón, para el desarrollo e implementación de un programa de DN. De esta capacitación participaron dos médicas pediatras, una de la 1ra. RS y otra de la $7 \mathrm{ma}$. RS, las cuales siguen trabajando en la red del PPFQRM y en algunos casos apoyando el tratamiento de los afectados (12).

A nivel de los STM, la capacitación para la apertura de un STM fue realizada de forma presencial para el $100 \%$ de los sitios, con la entrega de materiales, como la Guía para Toma de Muestra del Test del Piecito (13). En algunos casos, la capacitación fue apoyada por aquellos sitios que ya contaban con años de actividad.

En la Figura 3 se muestra el algoritmo de trabajo del PPFQMR, donde se visualizan los RRHH que intervienen, desde la toma de muestra hasta la entrega del resultado del RN.

Un caso se inicia con la toma de muestra del RN y termina con la entrega de un resultado negativo, lo cual se realiza en el STM donde la muestra ha sido extraída. Ante un resultado positivo, se procede a la búsqueda activa del RN de manera a iniciar el tratamiento de manera oportuna. 


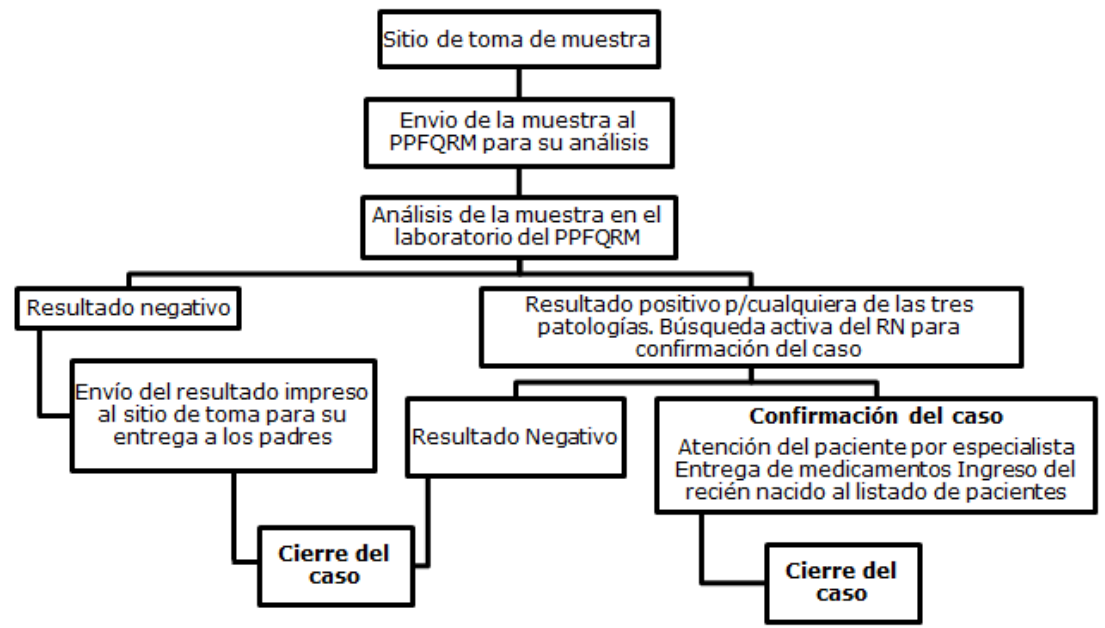

Figura 3. Algoritmo de trabajo del PPFQRM

Difusión: Se realiza a través de la entrega de dípticos y materiales de difusión impresos, se cuenta con un corto publicitario en Guaraní, Español y en Lenguaje de Señas. A su vez se cuenta con una página web, desde donde pueden bajarse todos los materiales impresos elaborados tanto para la difusión como para la capacitación de los padres y profesionales en las patologías atendidas (http://www.mspbs.gov.py/piecito/). A fin de llegar a las poblaciones blanco, que son las embarazadas y progenitores en edad fértil (Piecito y Amo mi bebé), se cuenta además con dos páginas en Facebook (https://www.facebook.com/piecito.mspybs; https://www.facebook.com/pages/Amo-mi -Bebe-pido-que-le-hagan-el-Test-Del-Piecito) (14-16).

Efectores para la recolección de muestras de sangre: En el $89 \%$ de los STM la persona encargada de la toma de muestra era un profesional de enfermería y el $11 \%$ restante correspondía a profesionales de obstetricia, medicina y bioquímica (Figura 4).

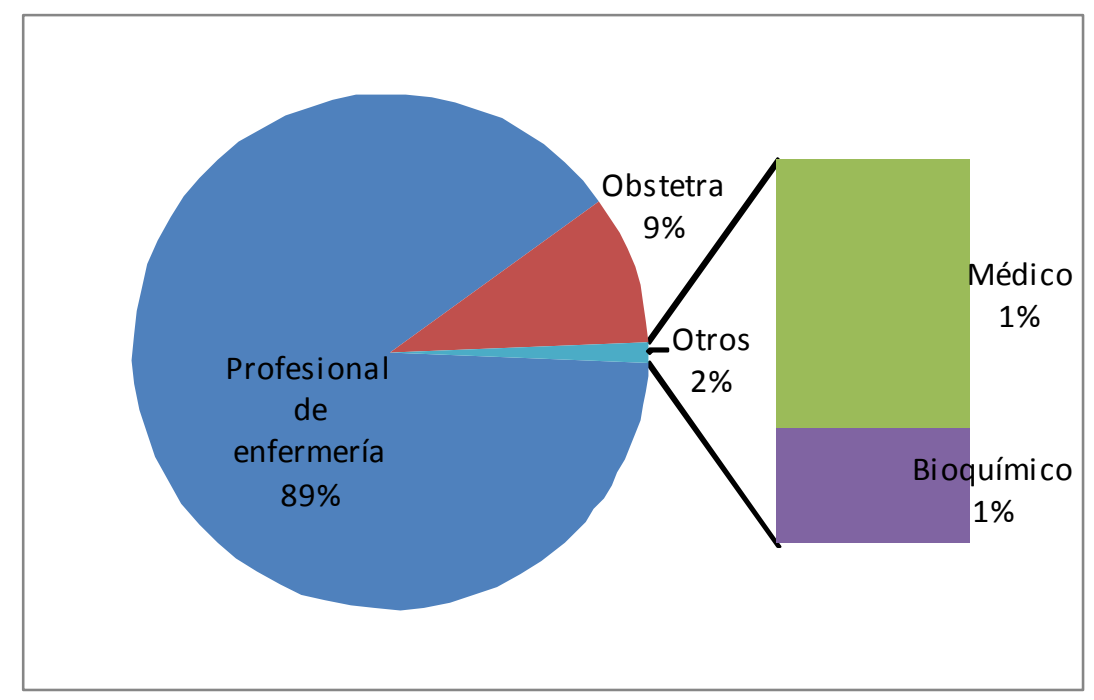

Fuente: Programa de Prevención de la Fibrosis Quística y Retardo Mental

Figura 4. Personal encargado de la toma de muestra en los STM 
Características de las muestras: Las muestras de los RN para los estudios de detección neonatal corresponden a gotas de sangre entera, obtenidas por punción del talón y recogidas en papel de filtro. Se utilizan éstas por la posibilidad del traslado vía correo, sin muchos inconvenientes para la conservación. Se utiliza suero para los pacientes con HC para los estudios de TSH y T4 mientras que para los pacientes con PKU se determina la FA en sangre entera recogida en papel de filtro. La fibrosis quística se determina por la tripsina inmunoreactiva.

Transporte: De los 942 STM registrados en el 2.014, el 65\% de los mismos envió sus muestras a través del correo nacional, con el cual se tiene un convenio de trasporte gratuito, un 33\% contaba con una persona encargada del traslado de las muestras hasta el PPFQRM y un $2 \%$ utilizó el correo privado para tal efecto, esto en especial ocurre con los hospitales que se encuentran en la Región del Chaco Paraguayo (Figura 5).

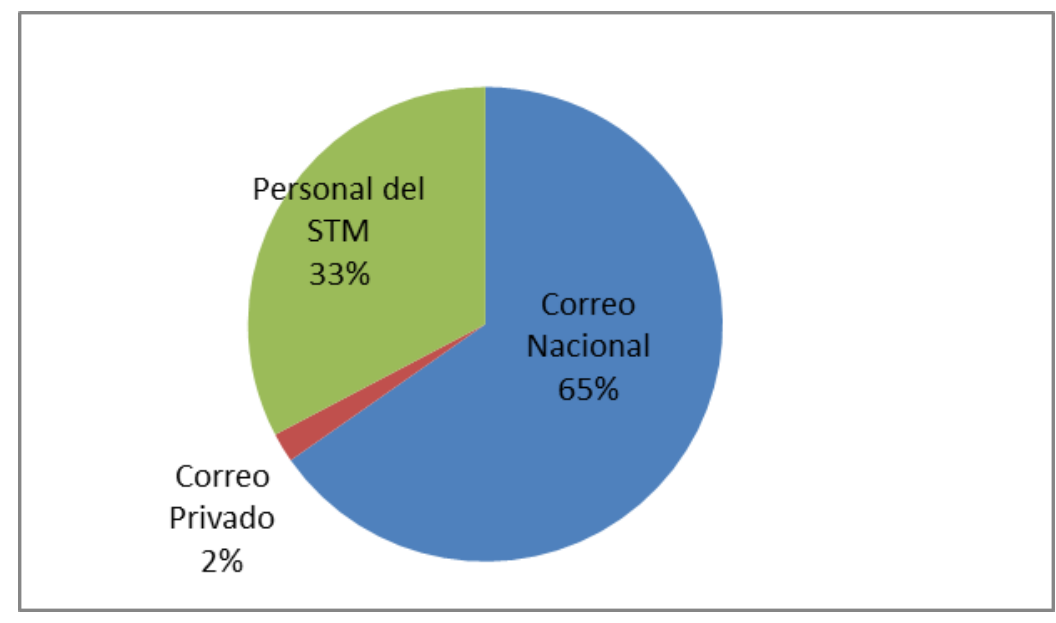

Fuente: Programa de Prevención de la Fibrosis Quística y Retardo Mental

Figura 5. Forma de envío de las muestras desde los STM. $n=942$

Laboratorio de pesquisa y confirmación: El proyecto piloto se inició en 1.999 con la detección del $\mathrm{HC}$ a través de la determinación de la Hormona Estimulante de la Tiroides (TSH) por el método de ELISA y la detección de la fenilcetonuria por el método microbiológico de Guthrie. En el 2.006, un equipo de fluorometría fue recibido en donación y se incorporaron reactivos de alta sensibilidad y especificidad, considerados líderes en el mercado. Esto ha permitido realizar el estudio a los RN antes del alta, un punto muy importante ya que en nuestro país, especialmente en el área rural los RN y sus madres son dados de alta a las 24 horas de vida y solo un $50 \%$ de las madres vuelve antes de los un mes de vida para su estudio. A su vez con la recepción en el 2.008 de otra donación, esta vez de un equipo para el estudio del test del sudor, se incorporó el estudio de la FQ de forma selectiva, dirigido a los parientes de personas fibroquisticas y otros RN con algún antecedente prenatal o síntomas perinatales de una fibrosis quística. Desde el proyecto piloto las muestras fueron procesadas en su totalidad para detectar el $\mathrm{HC}$ y la PKU. En el 2.006 se incorporó, como un proyecto piloto, el estudio de la FQ a través de la determinación de la Tripsina Inmuno Reactiva (IRT), realizándose este estudio en $30 \%$ de las muestras recibidas. Con el correr de los años el número de muestras procesadas fue aumentando y partir del 2.015, la IRT es determinada en todas las muestras ingresadas.

Todas las muestras provenientes de los 942 STM distribuidos en las 18 RS son trasladadas al laboratorio de pesquisa y confirmación que se encuentra ubicado en 
Asunción, capital del país.

Tratamiento: Los Artículos 1 y 7 de la Ley 2.138/2003 garantizan el tratamiento de los RN afectados. Los casos positivos son localizados a través de la búsqueda activa de la cual participan además de los miembros del nivel central del Programa, los encargados de los STM. En primer lugar se contacta con la familia a través del número de celular que figura en la hoja de toma de muestra, o la visita del encargado del STM. El RN es trasladado hasta Asunción para la extracción de una nueva muestra, para la realización de los estudios confirmatorios, en algunos casos, la madre es acompañada por un personal del STM. Esto, por lo general ocurre con aquellas madres guaraní parlantes y con las comunidades indígenas, donde dicha función es ejercida por el promotor de salud.

El tratamiento de HC consiste en la atención por una especialista en endocrinología, los estudios laboratoriales de TSH y T4 y la entrega de la levotiroxina sódica, así como en algunos casos de otros medicamentos como el sulfato de zinc o sulfato ferroso.

De los 296 RN detectados de octubre de 1.999 a diciembre del 2.014, el 95\% de los casos vino al PPFQRM para su tratamiento. El 5\% restante sigue su tratamiento en el lugar de residencia (doce casos) o solo viene al nivel central cuando es necesario el control laboratorial para el ajuste de la dosis de la levotiroixina. Tres RN fallecieron. Estos dos últimos grupos corresponden a personas de escasos recursos, grupos de indígenas o donde el traslado es muy oneroso y/o difícil.

El tratamiento de la fenilcetonuria, se lleva a cabo a través de la atención por una especialista en gastroenterología, quién a su vez tiene a su cargo el control de la ingesta diaria de proteínas para ajustar la dieta, debido a que no se cuenta con una nutricionista que apoye el tratamiento. Se hace entrega de una leche libre de fenilalanina, en la cantidad que recomendada por la especialista. También son entregados sulfato de zinc y sulfato ferroso para el buen crecimiento de los individuos en tratamiento. El control de la cantidad la FA en sangre se realiza a nivel laboratorial, ya sea de forma periódica, según corresponda a una PKU clásica o a una hiperfenilalaninemia, los padres son instruidos para que realicen la toma de muestra en casa y su posterior envío al PPFQRM para su análisis, junto con la lista de alimentos ingeridos desde el último control. El $100 \%$ de los pacientes afectados sigue su tratamiento en el PPFQRM.

Por último se informa sobre el tratamiento de la FQ, ésta patología también es atendida por la especialista en gastroenterología, siendo el aparato gastrointestinal uno de los más afectados, no se cuenta con especialista en neumología, pero si un kinesiólogo. A partir del 2.008 se hace entrega de las enzimas pancreáticas, sulfato de zinc, sulfato ferroso, azitromicina, salbutamol. Así como la entrega de la tobramicina, cuyo costo oscila los 4.500 dólares. También se entregan tapaboca y equipos de nebulización e incentivadores de la tos, estos últimos en especial a los grupos de escasos recursos económicos.

\section{Ejes o dimensiones de la cobertura universal en la DN}

Amplitud: En relación a los tres ejes o dimensiones de la cobertura según el modelo conceptual de la CUS propuesto por la OMS, el primer eje es la amplitud que corresponde a la proporción de la población cubierta. Para el análisis de amplitud se consideró solamente el número total de nacidos vivos en el 2.013 ya sea en instituciones públicas, privadas o en su domicilio cuyo nacimiento fue registrado. Así la amplitud, según el número de RN atendidos sobre el número de RN registrados, tenemos que en el 2.013 se estudió al $68,6 \%(73.398 / 106.946)$, mientras que en el 2.014 se alcanzó al $76,1 \%(85.000 / 111.677)(17,18)$. Tabla 2.

Estos porcentajes podrían ser mayores ya que al considerar el número total de nacidos vivos se incluye a los nacidos en el Instituto de Previsión Social (IPS) que es el seguro 
de los trabajadores del sector privado, así como a los nacidos en hospitales privados. En estos sitios se realiza también la DN, pero no se han podido contar con los datos para este análisis.

Tabla 2. Eje amplitud de la Cobertura Universal. Periodo 2.000-2.014

\begin{tabular}{llllll}
\hline Años & $\begin{array}{l}\text { Nacimientos } \\
\text { estimados }\end{array}$ & $\begin{array}{l}\text { Cobertura con } \\
\text { estimados }\end{array}$ & $\begin{array}{l}\text { Nacimientos } \\
\text { registrados }\end{array}$ & $\begin{array}{l}\text { Cobertura con } \\
\text { registrados }\end{array}$ & RN tamizados \\
\hline 2000 & 149.970 & 0,4 & 86.000 & 0,7 & 613 \\
2001 & 150.407 & 0,6 & 83.919 & 1,1 & 922 \\
2002 & 150.844 & 1,4 & 90.085 & 2,3 & 2051 \\
2003 & 151.281 & 3,0 & 86.739 & 5,2 & 4473 \\
2004 & 151.718 & 2,2 & 101.000 & 3,3 & 3367 \\
2005 & 152.155 & 12,4 & 105.808 & 17,8 & 18841 \\
2006 & 152.336 & 13,1 & 102.109 & 19,6 & 20002 \\
2007 & 152.517 & 12,5 & 95.862 & 19,9 & 19042 \\
2008 & 152.698 & 23,9 & 99.688 & 36,6 & 36520 \\
2009 & 152.879 & 28,8 & 102.162 & 43,2 & 44095 \\
2010 & 153.060 & 36,2 & 101.153 & 54,7 & 55370 \\
2011 & 153.012 & 43,8 & 105.825 & 63,4 & 67053 \\
2012 & 152.964 & 47,9 & 108.401 & 67,6 & 73293 \\
2013 & 152.916 & 48,0 & 106.946 & 68,7 & 73428 \\
2014 & $*$ & & 111.677 & 76,1 & 85.000 \\
\hline
\end{tabular}

Fuente: Programa de Prevención de la Fibrosis Quística y Retardo Mental.

*Los datos aun no están disponibles

En la Tabla 3, se presenta la cobertura por región sanitaria. Se observa una cobertura mediana de $67,3 \%$. En algunas regiones sanitarias, se obtuvieron coberturas por encima del $100 \%$ como en XVII y XVIII. La XVII RS corresponde al Departamento de Alto Paraguay y este nivel de cobertura podría corresponder al subregistro, pero los RN son captados para su estudio por las USF. En relación a la XVIII RS, está corresponde a la capital Asunción y esta cobertura sería debida al número de RN que vienen a la capital para tener a sus hijos, pero que vuelven y son registrados en su lugar de origen.

Para calcular el número de RN que podría no haber sido detectado, se utilizó el dato de incidencia para el 2.013, que para el $\mathrm{HC}$ es de $1 / 2.447$, con lo cual se tiene que 14 RN podrían haber nacido con $\mathrm{HC}$ y a su vez unos 5 con PKU, con la incidencia de 1/6.673. Es importante acotar que el número total registrado de 106.946 RN, corresponde a los nacidos en el IPS y seguros privados, donde los casos de HC son atendidos. Por lo tanto, esta brecha podría ser menor, lo cual no es igual para la PKU, pues está patología solo es tratada en el PPFQRM.

De los 33 casos detectados en el 2.014 para HC, 32 están durante el 2.015 bajo tratamiento. En relación a la PKU, el 100\% (9/9) de los casos iniciaron y están bajo tratamiento. En relación al número de RN que podría no haber sido detectado en el 2.014 , teniendo en cuenta la incidencia para ese año se esperaba detectar 44 nuevos de HC y 16 para la PKU. Por tanto en el 2.014 once casos de HC no fueron detectados o lo fueron fuera del PPFQRM y 7 casos con PKU, esta es la pérdida que ocurre cuando la cobertura no es aún universal. 
Tabla 3. Análisis de amplitud por RS según datos registrados. Año 2.013

\begin{tabular}{llllll}
\hline \multirow{2}{*}{ RS } & \multicolumn{2}{c}{ Población } & \multicolumn{2}{c}{ Casos detectados } \\
& Registrada & Tamizada & Cobertura (\%) & HC & PKU \\
\hline I & 4.178 & 2.645 & 63,3 & 3 & 0 \\
II & 6.605 & 4.218 & 63,9 & 1 & 0 \\
III & 4.146 & 2.789 & 67,3 & 0 & 0 \\
IV & 2.973 & 2.103 & 70,7 & 0 & 0 \\
V & 7.750 & 5.253 & 67,8 & 1 & 2 \\
VI & 2.375 & 1.427 & 60,1 & 1 & 0 \\
VII & 7.128 & 5.402 & 75,8 & 1 & 0 \\
VIII & 1.814 & 1.537 & 84,7 & 0 & 0 \\
IX & 3.028 & 2.232 & 73,7 & 0 & 0 \\
X & 15.069 & 9.220 & 61,2 & 3 & 1 \\
XI & 31.760 & 20.426 & 64,3 & 14 & 3 \\
XII & 935 & 789 & 84,4 & 0 & 1 \\
XIII & 2.811 & 1.224 & 43,5 & 0 & 0 \\
XIV & 3.048 & 1.813 & 59,5 & 1 & 0 \\
XV & 1.901 & 676 & 35,6 & 2 & 0 \\
XVI & 1.314 & 159 & 12,1 & 0 & 0 \\
XVII & 289 & 837 & 289,6 & 0 & 0 \\
XVIII & 9.365 & 10.648 & 113,7 & 3 & 4 \\
País & 106.946 & 73.398 & 68,6 & 30 & 11 \\
\hline
\end{tabular}

Fuente: Programa de Prevención de la Fibrosis Quística y Retardo Mental. RS: Región Sanitaria; HC: hipotiroidismo congénito; PKU: fenilcetonuria

La incidencia de HC y PKU para el 2.014 y la incidencia acumulada desde 1.999 a diciembre del 2.014 se muestran en la Tabla 4 siendo $1 / 2.576$ para la primera y 1/9.444 para la segunda.

Tabla 4. Incidencia acumulada y puntual del HC y la PKU. Periodo 1.999-2.014

\begin{tabular}{lcccc}
\hline & \multicolumn{2}{c}{ Año 2.014 } & \multicolumn{2}{c}{ Octubre 1.999 a diciembre 2.014 } \\
& HC & PKU & HC & PKU \\
\hline No de casos detectados/ & $33 / 85.000$ & $9 / 85.000$ & $296 / 504.211$ & $56 / 504.211$ \\
No de RN atendidos & $1 / 2.576$ & $1 / 9.444$ & $1 / 1.703$ & $1 / 9.004$ \\
Incidencia & & & & \\
\hline
\end{tabular}

En la Tabla 5, se visualiza el número de STM por RS en el 2013, en relación al número de distritos que se mantiene constante de 246, al número de habitantes y al número de establecimientos de salud. En relación a este último grupo se cuenta con 17 Hospitales Regionales, uno por RS; 37 Hospitales Distritales; 11 Hospitales Materno Infantil; 557 Centros y Puestos de Salud y 781 Unidades de Salud Familiar (USF). De la misma manera que a nivel del MSPyBS la diferencia en número de establecimientos de salud entre un año y otro está dada por la apertura de nuevas USF, en el PPFQRM la diferencia 
entre un año y otro se da en base a la apertura de nuevos STM en las USF, habiéndose cubierto en principio los hospitales anteriormente citados.

Tabla 5. No de $\mathrm{HC}$ y PKU por $\mathrm{N}^{\circ}$ de STM, RS en relación al $\mathrm{N}^{\circ}$ de Distritos y de habitantes. Año 2.013

\begin{tabular}{|c|c|c|c|c|c|}
\hline RS & No de STM & No de Distritos & No de habitantes & $\begin{array}{l}\mathrm{N}^{\circ} \mathrm{de} \\
\mathrm{HC}\end{array}$ & $\begin{array}{l}\text { No de } \\
\text { PKU }\end{array}$ \\
\hline I & 48 & 9 & 189.660 & 10 & 0 \\
\hline II & 55 & 20 & 361.513 & 16 & 0 \\
\hline III & 53 & 20 & 285.998 & 12 & 1 \\
\hline IV & 56 & 18 & 198.523 & 10 & 0 \\
\hline v & 60 & 22 & 484.177 & 10 & 3 \\
\hline VI & 42 & 11 & 151.477 & 5 & 1 \\
\hline VII & 87 & 30 & 551.128 & 11 & 1 \\
\hline VIII & 30 & 10 & 119.719 & 5 & 1 \\
\hline IX & 44 & 17 & 239.661 & 6 & 0 \\
\hline$x$ & 88 & 22 & 801.790 & 18 & 4 \\
\hline$X I$ & 65 & 19 & 2.297 .739 & 110 & 13 \\
\hline XII & 41 & 16 & 84.268 & 4 & 2 \\
\hline XIII & 8 & 4 & 125.746 & 0 & 1 \\
\hline XIV & 35 & 12 & 195.336 & 6 & 2 \\
\hline$x V$ & 18 & 8 & 108.520 & 2 & 1 \\
\hline XVI & 13 & 4 & 62.784 & 0 & - \\
\hline XVII & 7 & 3 & 11.057 & 4 & 1 \\
\hline XVIII & 22 & 1 & 514.267 & 34 & 15 \\
\hline Total & 772 & 246 & 6.783 .363 & 263 & 46 \\
\hline
\end{tabular}

Fuente: Programa de Prevención de la Fibrosis Quística y Retardo Mental

Eje profundidad: este eje fue analizado a través de la comparación de las patologías que son cubiertas a nivel de países en Latinoamérica o de otros países. Además se evaluaron las patologías detectadas en otras instituciones a nivel nacional como el IPS y los servicios privados (Tabla 6).

Tabla 6. Eje profundidad a través de las patologías cubiertas por el programa de DN en el Paraguay

\begin{tabular}{llll}
\hline $\begin{array}{l}\text { Patologías incluidas } \\
\text { en Latinoamérica }\end{array}$ & $\begin{array}{l}\text { Patologías } \\
\text { detectadas en el } \\
\text { PPFQRM }\end{array}$ & $\begin{array}{l}\text { Patologías } \\
\text { detectadas en IPS }\end{array}$ & $\begin{array}{l}\text { Patologías } \\
\text { detectadas a nivel } \\
\text { privado }\end{array}$ \\
\hline $\mathrm{HC}$ & $\mathrm{HC}$ & $\mathrm{HC}$ & $\mathrm{HC}$ \\
$\mathrm{PKU}$ & $\mathrm{PKU}$ & $\mathrm{PKU}$ & $\mathrm{PKU}$ \\
$\mathrm{FQ}$ & & $\mathrm{FQ}$ \\
$\mathrm{GAL}$ & $\mathrm{FQ} *$ & & $\mathrm{GAL}$ \\
$\mathrm{HSC}$ & $\mathrm{GAL**}$ & & $\mathrm{HSC}$ \\
\hline
\end{tabular}

Fuente: PPFQRM.

*En el 2.013 se analizaba solo el $30 \%$ de las muestras y a partir del 2.015 el $100 \%$.

**Patologías con proyectos pilotos en curso. 
Otro punto importante a la hora de evaluar la profundidad de la CUS, son los métodos de detección para el análisis de las muestras, así como el esquema terapéutico utilizado y el recomendado. En la Tabla 7, se presentan los métodos de detección así como el esquema de tratamiento utilizados ya sea en el programa nacional o en el seguro de IPS.

Tabla 7. Eje profundidad a través de los métodos de detección y esquemas terapéuticos

\begin{tabular}{llll}
\hline Patología & & $\begin{array}{c}\text { Método de } \\
\text { detección }\end{array}$ & \multicolumn{1}{c}{ Esquema terapéutico utilizado y recomendado } \\
\hline HC & $\begin{array}{l}\text { PPFQRM } \\
\text { IPS }\end{array}$ & $\begin{array}{l}\text { Fluorométrico } \\
\text { ELISA }\end{array}$ & $\begin{array}{l}\text { Atención por especialista, seguimiento hormonal y en- } \\
\text { trega de medicamentos. }\end{array}$ \\
PKU & PPFQRM & Fluorométrico & $\begin{array}{l}\text { Atención por especialista, seguimiento laboratorial y } \\
\text { entrega de leche libre de fenilalanina }\end{array}$ \\
& IPS & ELISA & $\begin{array}{l}\text { No ofrece tratamiento } \\
\text { Atención por especialistas y entrega de medicamentos, } \\
\text { incluida la tobramicina, no incluye la alfadornasa. }\end{array}$ \\
\hline
\end{tabular}

Fuente: Programa de Prevención de la Fibrosis Quística y Retardo Mental

Expansión potencial: A nivel mundial la expansión de los programas de DN, se realiza teniendo en consideración los criterios de Wilson y Junger (19). El proyecto piloto se inició para los trastornos auditivos en noviembre del 2.012 y en curso están los proyectos para la galactosemia y la hiperplasia suprarrenal congénita, contemplados en el Panel Ampliado así como los estudios por Espectrometría de Masa en Tándem, que incluye el concepto de un análisis la detección o sospecha de 30 posibles patologías así como los estudios neonatales de visión y de cardiopatías. En la Tabla 8 se ven los tipos de paneles de DN, así como las patologías que los pueden llegar a componer $(4,19)$.

Grado de protección financiera: En el Artículo 3 de la Ley 2138/2003, se estipula que el presupuesto para llevar adelante el PPFQRM será contemplado en el Presupuesto General de la Nación. Con él se cubre el pago a todo el personal del nivel central y la compra de los reactivos, insumos y medicamentos así como el combustible para las capacitaciones de los STM, viáticos y la impresión de los materiales de toma de muestra. Por otro lado, desde el proyecto piloto hasta la actualidad, el PPFQRM ha contado siempre con el apoyo de organismos como la Itaipu Binacional, la UNICEF, la JICA, para las actividades de capacitación, difusión, equipamiento, entre otras necesidades eventualmente no cubiertas por el presupuesto del Programa.

Un dato importante de mencionar en relación al grado de protección financiera, es que el PPFQRM desde su inicio y a lo largo de todos estos años no ha sido descontinuado por falta de reactivo ni de medicamentos para el tratamiento. Si ha debido de instaurar la detección de manera selectiva para los análisis de la FQ, debido a que el tratamiento es altamente costoso y se debe medir la incorporación de nuevos casos.

Estrategias identificadas para lograr la CUS: Entre las estrategias identificadas para lograr la anhelada cobertura universal, se pueden citar: 1) Aumento de los STM, a fin de captar en especial a los grupos más vulnerables, minimizando la brecha entre las instituciones de salud y los STM, 2) Tareas de sensibilización dirigida a los políticos, a fin de que asegurar el presupuesto del PPFQRM, 3) Inversión en la infraestructura del PPFQRM, a fin de asegurar la calidad de los estudios ofrecidos, 4) Aumento de la canasta de medicamentos e insumos entregados, 5) Incorporación de los RRHH que faltan para llevar adelante las actividades del Programa de DN, 6) Organizar redes de 
atención en los Hospitales que cuentan con profesionales que pueden apoyar en el tratamiento de los pacientes detectados, 7) Revisar la Ley 2138 del 2.003, que creó el PPFQR, a fin de incluir una mayor cobertura así como más patologías a pesquisar.

Tabla 8. Patologías incluidas en los tres tipos de paneles de detección neonatal

\begin{tabular}{llll}
\hline & Panel & & \\
\hline Patologías incluidas & Básico & Ampliado & Expandido \\
Hipotiroidismo congénito & $\mathrm{x}$ & $\mathrm{x}$ & $\mathrm{x}$ \\
Fenilcetonuria & $\mathrm{x}$ & $\mathrm{x}$ & $\mathrm{x}$ \\
Fibrosis Quística & $\mathrm{x}$ & $\mathrm{x}$ & $\mathrm{x}$ \\
Galactosemia & & $\mathrm{x}$ & $\mathrm{x}$ \\
Hiperplasia suprarrenal congénita & $\mathrm{x}$ & $\mathrm{x}$ \\
Hemoglobinopatías & & $\mathrm{x}$ & $\mathrm{x}$ \\
Deficiencia de Biotinidasa & $\mathrm{x}$ & $\mathrm{x}$ \\
Deficiencia de Glucosa 6 P-Deshidrogenasa & & $\mathrm{x}$ & $\mathrm{x}$ \\
Otras aminoacidopatías & & $\mathrm{x}$ \\
Defectos de la Oxidación de Ácidos grasos & & $\mathrm{x}$ \\
Acidurias orgánicas & & $\mathrm{x}$ \\
Inmunodeficiencia combinada severa & & $\mathrm{x}$ \\
Enfermedades de Almacenamiento Lisosomal & & $\mathrm{x}$ \\
HIV, Toxoplasmosis, Chagas & & $\mathrm{x}$ \\
Trastornos auditivos y de la visión & & $\mathrm{x}$ \\
Cardiopatías congénitas & & $\mathrm{x}$ \\
\hline
\end{tabular}

Fuente: Elaboración propia.

\section{DISCUSIÓN}

En el presente estudio se describe la situación actual del Programa Nacional encargado de la detección neonatal en Paraguay y se analizan las brechas para el logro de la cobertura universal. La cobertura en salud en el Paraguay está dada por la atención que se brinda a través de la red de instituciones del MSPyBS que divide al país en 18 Regiones Sanitarias correspondiendo a los 17 departamentos y Asunción, y cubre el $70,8 \%$ de la población del país. El seguro de los trabajadores en el Instituto de Previsión Social (IPS) cubre el 20,1\%, y los otros tipos de seguro el 9,1\% restante (20).

El Programa de detección neonatal nace como un proyecto piloto de investigación para prevenir el retardo mental producido por el HC en 1.999. A partir de ese año ha ido creciendo con el apoyo constante de organizaciones nacionales e internacionales. Con la Ley No 2.138/2003 se creó el Programa de Prevención de la Fibrosis Quística y del Retardo Mental (PPFQRM), y se responsabilizó al Ministerio de Salud Pública y Bienestar Social para su planificación y ejecución, con los fondos del Presupuesto General de la Nación (21). 
El programa actualmente cuenta con 942 sitios de toma de muestra distribuidos en todas las regiones sanitarias. Cada sitio cuenta con por lo menos dos personas. La apertura de nuevos STM es una de las estrategias más válidas para lograr la CUS, teniendo en cuenta que en el 2.013 se llegó a un $55 \%$ del total de instituciones de salud y para el 2.014 se tuvo un aumento de STM y a su vez del número de muestras que de 73.398, pasaron a 85.000, en especial con apertura en zonas de mayor pobreza.

Es imprescindible garantizar la sostenibilidad del financiamiento cuando se amplia y profundiza la cobertura. Para ello es necesario asegurar el presupuesto asignado así como también una gestión adecuada para movilizar los recursos que permitan brindar servicios integrales, continuos y oportunos. Si bien desde su inicio y a lo largo de todos estos años no hubo discontinuidad, es necesario señalar que los costos elevados que suponen la inclusión de nuevas patologías pueden traducirse en una implementación progresiva más lenta, como ha ocurrido con la fibrosis quística.

La incidencia de HC y PKU para el 2.014 son de $1 / 2.576$ y $1 / 9.444$ respectivamente. Las incidencias de estas dos patologías se han mantenido estables desde 1.999 hasta la fecha. La incidencia de hipotiroidismo congénito en nuestro país es la más alta de la región. Argentina tiene un índice de 1 cada 2.500 nacidos vivos, en Uruguay y Brasil afecta a 1 de cada 3.000.Esto es debido a la mediterraneidad del país con terrenos pobres en yodo, esta deficiencia ha sido y debe seguir siendo considerada como un grave problema de salud, como factor contribuyente al nacimiento de niños y niñas con HC (21).

El tamizaje neonatal para la fenilcetonuria y el hipotiroidismo congénito son los mejores establecidos y aceptados, tanto en efectividad como en eficiencia en todo el mundo (22). En el análisis realizado en Canarias por Pérez Hernández se demostró que el beneficio neto y relación costo-beneficio del tamizaje neonatal para la fenilcetonuria y el hipotiroidismo fueron muy altos (23) mostrando que por cada peseta gastada se obtenía un beneficio de 9,40 ptas. Este resultado fue similar al reportado por el Centro Regional de Despistaje de Enfermedades metabólicas en la Infancia en Verdun, Francia con un ratio beneficio/costo de 13,8 para el hipotiroidismo congénito y de 6,6 para la fenilcetonuria (24). Otros países han mostrados incluso ratios más altos. Los mayores beneficios son los de evitar las cargas que para la sociedad y la familia suponen los costos de los niños con retraso mental $(25,26)$.

La cobertura universal en salud, definida por la Organización Mundial de la Salud (OMS) como el acceso universal a servicios de salud integrales a un costo razonable y sin riesgos financieros mediante la protección contra gastos catastróficos en salud fue evaluada en este estudio para el programa de detección neonatal (27).

En relación específicamente al Programa de detección neonatal en Paraguay el primer eje analizado fue el eje de la amplitud que fue definida por el porcentaje de RN tamizados en relación a la población registrada y la población estimada. Se observó un aumento altamente significativo desde su inicio en 1.999 en cuando solo se tenía una cobertura de $0,4 \%$ hasta llegar a $76,1 \%$ en el 2.014 . Esto demuestra los grandes esfuerzos realizados y la existencia de voluntad política de los decisores hacia la universalización de la cobertura en la detección neonatal en Paraguay.

Cuando se realizó el análisis de la cobertura por región sanitaria en el 2.014 se encontraron grandes diferencias entre ellas, de esta manera una región del Chaco Paraguayo tuvo una cobertura de $290 \%$, lo que implica que la toma de muestra que se realiza en esta región corresponde a la población de otras regiones sanitarias, lo mismo ocurre con la capital del país (114\%). Se tuvieron coberturas muy bajas en ciertas regiones lo que demuestra la existencia de brechas sustanciales entre distritos y departamentos.

Otro eje de la CUS analizado fue la profundidad que fue evaluada a través de las patologías cubiertas por el programa en comparación a las incluidas en los programas a nivel de los países de Latinoamérica y otras instituciones a nivel nacional. En otros 
países de Latinoamérica, son cinco las patologías que se incluyen en la detección neonatal y en el programa son tres. En Paraguay, a nivel de los servicios privados son detectadas las cinco mencionadas patologías pero solo una población muy limitada del país tiene acceso a ellas. A nivel mundial la expansión de los programas de DN se realiza teniendo en consideración los criterios de Wilson y Junger que ponen de manifiesto la necesidad de llevar adelante un proyecto piloto que de hecho ya inició para los trastornos auditivos en noviembre del 2.012 y se prevé el inicio de proyectos pilotos para la galactosemia y la hiperplasia suprarrenal congénita en el 2.015. Estos están contemplados en el Panel Ampliado así como los estudios por Espectrometría de Masa en Tándem, que incluye el concepto de un análisis la detección o sospecha de 30 posibles patologías así como los estudios neonatales de visión y cardiopatías. Estos últimos grupos aún son cuestionados debido a que no pueden implementarse de forma universal y tanto la efectividad como los beneficios de la detección y tratamiento precoz no siempre son positivos (28).

Otro punto importante a la hora de evaluar la profundidad de la CUS, son los métodos de detección para el análisis de las muestras, así como el esquema terapéutico utilizado y el recomendado. Vemos que tanto el método de detección como los tratamientos ofrecidos en el PPFQRM son los recomendados, por otro lado el seguro de IPS, considerado el de mayor cobertura entre los privados, no ofrece las tres patologías estipuladas por ley y en uno de los casos, si bien detecta la patología no contempla el tratamiento de la misma, de ahí que todos los casos que son detectados por otras instituciones son finalmente derivados al Programa para su tratamiento y seguimiento.

En relación al tercer eje del CUS, nivel de grado de protección financiera, en Paraguay en el Artículo 3 de la Ley 2138/2003, se contempla en el Presupuesto General de la Nación el pago a todo el personal del nivel central y la compra de los reactivos, insumos y medicamentos así como el combustible para las capacitaciones de los STM, viáticos y la impresión de los materiales de toma de muestra. Pero se hace necesario asegurar el presupuesto para que el aumento se dé de manera natural por año, más aún cuando la meta es lograr una cobertura universal. Si bien el Programa desde su inicio y a lo largo de todos estos años no ha sido descontinuado, el hecho de que la FQ, patología donde el tratamiento es de alto costo, haya debido implementarse de manera selectiva y su cobertura al total de muestra se haya demorado un quinquenio, no nos permite asegurar que de lograrse una cobertura universal esta pueda ser sostenible. De ahí, la importancia de sensibilizar y concientizar a las autoridades y en especial a aquellas responsables de otorgar el Presupuesto del Estado, asegurando de esta manera que no solo todos los servicios e insumos sigan siendo proveídos a todas aquellas personas que los demandan independientemente de su condición socioeconómica, con el arancel cero, eliminando el pago directo, sino la entrega de la canasta de medicamentos de calidad y en la cantidad necesaria. Este aspecto es vital para el programa considerando los determinantes sociales de salud que rodean a las personas afectadas por el $\mathrm{HC}$ en Paraguay que fuera descripta en un estudio realizado en 113 familiares de 114 pacientes quienes fueron entrevistados en relación a sus características socio-demográficas y situación laboral. Se observa que el $69,3 \%$ de los pacientes con $\mathrm{CH}$ era del sexo femenino, el $71,1 \%$ tenía menos de 5 años de edad, el $40,4 \%$ vivía en zonas rurales y un $81,6 \%$ no tenía seguro médico. En relación a la madre, el $72,6 \%$ de estas tenía entre 15 y 30 años al momento del nacimiento del hijo, un $38,1 \%$ con un nivel educativo primario y un $2,7 \%$ analfabeta, un $62,4 \%$ sin ingresos y $22 \%$ con empleo informal. La mediana del número de hijos fue 2 (rango: 1-11). Las características socio-demográficas como la inequidades identificadas, destacan la importancia de los programas preventivos de salud como medida positiva para la reducción de las consecuencias de los determinantes sociales tanto en las personas afectadas como en el núcleo familiar (29).

Por tanto al buscar la cobertura sanitaria universal, no solo se llegaría al $100 \%$ de los recién nacidos con condiciones discapacitantes, sino se reduciría la morbimortalidad en 
esta población, permitiéndole una vida normal tanto al individuo afectado como a sus familias y a la comunidad en la que aquel se desenvuelve, permitiendo romper el círculo de la pobreza que nos indica que la discapacidad genera pobreza y la pobreza discapacidad. La cobertura sanitaria universal significa entonces preocuparse por la realización efectiva del derecho de cada quien a la salud.

\section{REFERENCIAS BIBLIOGRÁFICAS}

1. Dye Ch, Boerma T, Evans D, Harries A, Lienhardt Ch, McManus $\mathrm{J}$, et al. Informe sobre la salud: Investigación para una cobertura sanitaria universal. 2013.

2. Organización Mundial de la Salud. La atención primaria de salud más necesaria que nunca. Informe sobre la salud en el mundo 2008: informe de un Grupo Científico de la OMS. Ginebra: OMS; 2008.

3. Bickel $H$. Rationale of neonatal screening for inborn errors of metabolism. In: Bickel $\mathrm{H}$, Guthrie R, Hammersen G, eds. Neonatal screening for inborn errors of metabolism. Berlín: Springer; 1980. p.1-6.

4. Therrell BL, Panny SR, Davidson A, Eckman J, Hannon WH, Henson MA, et al. U.S. Newborn screening system guidelines: Statement of the Council of Regional Networks for Genetic Services. Screening 1, (1992):135-47.

5. Pass KA, Lane PA, Fernhoff PM, Hinton CF, Panny SR, Parks JS, et al. U.S. Newborn screening system guidelines II: Follow-up of children, diagnosis, management, and evaluation statement of the Council of Regional Networks for Genetic Services (CORN). J Pediatr. 2000;137(4 Suppl):S146.

6. Organización Panamericana de la Salud. Redes integradas de servicios de salud: Conceptos, opciones de política y hoja de ruta para su implementación en las Américas. Washington DC: OPS; 2010. Serie: La Renovación de la Atención Primaria de la Salud en las Américas No 4.

7. Programa de Prevención de la Fibrosis Quística y del Retardo Mental. Marco legal: Ley No 2.138/2.003. /Internet/./(Acceso 30 Mar. 2.015). Disponible en: http:// www.mspbs.gov.py/piecito/marco-legal.

8. Amarilla Gundín M, Arena Ansótegui J, Arrizabalaga Abásolo JJ, Díaz-Cadórniga F, Donnay Candil S, Escobar del Rey F. Iodo y salud en el siglo XXI. Madrid: Editorial Médica; 2004.

9. Queiruba G, Lemes A, Ferola C, Machado M, Queijo C, Garlo P, et al. Pesquisa neonatal: Lo que puede prevenir una gota de sangre. Montevideo: BPS, Centro de Estudios en Seguridad Social, Salud y Administración; 2010.
10.Morales Pérez P, Sánchez Zapardiel E. Capítulo 1. Identificación, estructura y expresión del Gen CFTR. En: Salcedo Posadas A, Gartner S, Girón Moreno RM, García MD, editores. Tratado de fibrosis quística. Madrid: Editorial Justim; 2012. p.29-40.

11. Ministerio de Salud Pública y Bienestar Social, Dirección General de Programas de Salud, Programa de Prevención de Fibrosis Quística y del Retardo Mental. Organigrama: Fibrosis quística /Internet/. Asunción: Ministerio de Salud Pública y Bienestar Social; 2009. (Acceso 10 jun 2015). Disponible en: http://www.mspbs.gov.py/ piecito/wp-content/uploads/2015/06/guiaFibrosis_FINAL-ult30AGOSTO.pdf

12.Programa de Prevención de la Fibrosis Quística y del Retardo Mental. Informe de proyecto de reducción del retardo mental apoyado por la JICA. Asunción: Programa de Prevención de la Fibrosis Quística y del Retardo Mental; 2011.

13. Ascurra M, Valenzuela A, Blanco F, Ortíz L, Núñez A, Escobar J. Guía nacional de toma de muestra /Internet/. Lambaré: Ministerio de Salud Pública y Bienestar Social, Programa de Prevención de la Fibrosis Quística y del Retardo Mental; 2013. / acceso 30 Mar. 2015/. Disponible en: http://www.mspbs.gov.py/piecito/wpcontent/uploads/2015/06/GUIA CORREGIDA.compressed.pdf

14.Programa de Prevención de la Fibrosis Quística y del Retardo Mental. Videos sobre el Programa de Prevención de Fibrosis Quística y del Retardo Mental en español./ Videos/. /Internet/. / acceso 30 Mar.I 2015/. Disponible en: http:// www.mspbs.gov.py/piecito/videos/

15. Ministerio de Salud Pública y Bienestar Social. Piecito Mspybs está en Facebook. / Internet/. /acceso 30 Mar. 2015/. Disponible en: https://www.facebook.com/ piecito.mspybs

16. Ministerio de Salud Pública y Bienestar Social. Amo $\mathrm{mi}$ bebe pido el Test del Piecito /Internet/. /acceso 30 Mar 2015/. Disponible en: https://www.facebook.com/ testdelpiecito. 
17.Dirección General de Estadística Encuestas y Censos (DGEEC). Proyección de población por departamentos según sexo y grupos de edad. Período 2000-2050: Subsistema de información de estadísticas vitales. Fernando de la Mora: DGEEC; 2005.

18. Ministerio de Salud Pública y Bienestar Social, Dirección General de Información Estratégica en Salud, Dirección de Estadística en Salud. Nacidos vivos por sitio de nacimiento según región sanitaria de residencia año 2014. Asunción: Dirección General de Información Estratégica en Salud, Dirección de Estadística en Salud; 2015.

19.Borrajo GJC. Pesquisa neonatal de enfermedades congénitas/Tesis Doctoral/. Universidad Nacional de la Plata. La Plata: Facultad de Ciencias Exactas. Departamento de Biología; 2011.

20.Dirección General de Estadística Encuestas y Censos (DGEEC). Salud. En: Principales resultados: Encuesta permanente de hogares 2013. Fernando de la Mora: DGEEC; 2014. P.23-6.

21.Ascurra M, Ayala A, Rodríguez S, Cabrera T, Covis L, Jara York J, et al. Programa de prevención del retardo mental en Caacupé [resumen]. Pediatr. (Asunción). 2001; 28 (1):33-6.

22. World Health Organization. Assessment of iodine deficiency disorders and monitoring their elimination: A guide for programme managers. 2nd ed. Geneva: World Health Organization; 2001.
23.Pérez Hernández R. Análisis coste-beneficio del programa de "screening" neonatal en Canarias/Tesis doctorales/. Canarias: Universidad de La Laguna; 2003.

24.Dhont JL, Farriaux JP, Sailly JC, Lebrun T. Economic evaluation of cost-benefit ratio of neonatal screening procedure for phenylketonuria and hypotiroidism. J Inher Metab Dis. $1991 ; 14: 633-9$.

25. Hisashige A. Health economic analysis of the neonatal screening program in Japan. Intl J Technol Asses Health Care.1994;10:382-91.

26.Barden HS, Kessel R, Schuett VE. The costs and benefits of screening for PKU in Wisconsin. Soc Biol. 1984;31(1-2):1-17.

27.WHO. Health systems financing the path to universal coverage. Geneva: World Health Organization; 2010.

28.Paz Valiñas L, Atienza Merino G. Efectividad clínica del cribado neonatal de los errores congénitos del metabolismo mediante espectrometría de masas en tándem. Revisión sistemática /Internet/. Madrid: Ministerio de Sanidad y Consumo; 2007. / acceso 30 Mar. 2015/. Disponible en: h t t p : / / a un ets. is ci i i.e s/ f i c h e rosproductos/110/ Informetandem.pdfAsunción:

29.Ascurra M, Samudio M, Escobar J. Determinantes sociales de salud en personas afectadas por el hipotiroidismo congénito en Paraguay. Pediatr (Asunción). 2014; 41(Supl): 81. 